\title{
Recent progress in theory of nonlinear pulse propagation in optical fibers
}

\author{
(Invited Paper)
}

\author{
Shalva Amiranashvili \\ and Uwe Bandelow \\ Weierstrass Institute for \\ Applied Analysis and Stochastics \\ Mohrenstraße 39, 10117 Berlin, Germany \\ Shalva.Amiranashvili@wias-berlin.de \\ Uwe.Bandelow@wias-berlin.de
}

\author{
Nail Akhmediev \\ Optical Sciences Group \\ Research School of Physics and Engineering \\ Institute of Advanced Studies \\ The Australian National University \\ Canberra ACT 0200, Australia \\ nna124@rsphysse.anu.edu.au
}

\begin{abstract}
We review recent achievements in theory of ultrashort optical pulses propagating in nonlinear fibers. The following problem is especially emphasized: what is the shortest duration (the highest peak power) of an optical soliton and which physical phenomenon is responsible for breakdown of too short pulses. We argue that there is an universal mechanism that destroys sub-cycle solitons even for the most favorable dispersion profile.
\end{abstract}

\section{PROBLEM POSING}

Extremely intense and extremely short optical pulses, in particular, stable few-cycle optical solitons, are important for many fundamental and applied aspects of modern nonlinear optics [1]-[3]. Such pulses have special properties and require special tools for their mathematical description. To a large extent pulse properties are captured by a properly generalized nonlinear Schrödinger equation, the latter can yield an appropriate complex field envelope even on a single cycle level [4]-[6]. On the other hand, one can describe an ultrashort pulse in terms of a more special short-pulse-equation. Several non-envelope short-pulse-equations were developed recently to deal with the special regimes of pulse propagation [7]-[17]. In this contribution we review recent progress in this quickly developing field.

A problem of special interest is the following question: what is the shortest possible soliton duration? Or, in other words, what is the largest possible peak power that is still compatible with the stable regime of pulse propagation? We address this problem by analyzing different equations for short pulses. Solitary waves appear as continuous families of solutions. As pulse duration decreases, the shape of the soliton becomes more and more steep and finally approaches the shortest shape with a cusp point at the top. Such behavior was observed for several short-pulse-equations with different origins. We argue that this is an universal feature.

To check our assumptions we analyzed the problem of the shortest soliton directly on the field level using a proper reformulation of Maxwell equations with the cubic nonlinear term and arbitrary dispersion. The tendency to cusp formation was observed also in this general case. As opposed to idealized short-pulse-equations, the soliton can be destroyed before it achieves the shortest possible duration. This happens when significant part of the soliton spectrum belongs to the normal dispersion domain. In such a case the soliton is destroyed by the higher-order dispersion effect, usually due to the so-called Cherenkov radiation [18].

\section{Methods}

Solitary solutions of different short-pulse-equations often appear as homoclinic trajectories of a reduced dynamical system with a simple interpretation: we deal with "energy conservation" in an imaginary mechanical system

$$
\frac{1}{2}\left(\phi^{\prime}\right)^{2}+U(\phi)=\text { const. }
$$

Here $\phi$ and $\phi^{\prime}$ are the field in question and its derivative. The effective potential $U(\phi)$ depends on the choose of the shortpulse-equation and on additional parameters such as pulse duration. As the latter decreases the potential evolves (Fig. 1a) and demonstrates singular behavior. Namely, a harmless singularity (Fig. 1a, green line) is finally replaced by a critical one (brown line) that destroys the soliton. This phenomenon was found both for several available short-pulse-equations [19][21] and for the generalized NLSE [22]. The "very last" (i.e., the shortest) soliton (Fig. 1a, red effective potential) yields a cusp shape.

Cusp existence was also confirmed in a general setting by direct numerical solution of Maxwell equations for several typical dispersion laws. We use a non-envelope bidirectional nonlinear wave equation with the cubic nonlinearity and arbitrary dispersion. The complex formulation naturally separates contributions of the first and the third harmonics, the latter is neglected. The propagation equation reads [23]

$$
\partial_{z}^{2} \mathcal{E}-\frac{1}{c^{2}} \partial_{t}^{2}\left(\hat{\epsilon} \mathcal{E}+\frac{3}{4} \chi^{(3)}|\mathcal{E}|^{2} \mathcal{E}\right)=0
$$

where

$$
\hat{\epsilon}\left(\sum_{\omega} \mathcal{E}_{\omega} e^{-i \omega t}\right)=\sum_{\omega} \epsilon(\omega) \mathcal{E}_{\omega} e^{-i \omega t},
$$

and to a good approximation the dielectric function $\epsilon(\omega)$ is purely real in the fiber transparency region. The real-valued electric field $E(z, t)=\operatorname{Re}[\mathcal{E}(z, t)]$. 

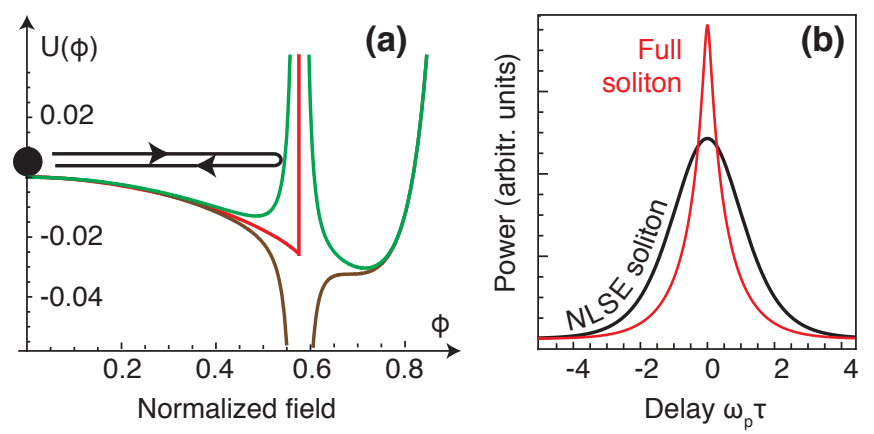

Fig. 1. (a) Effective potentials calculated for different durations of the resulting optical soliton are shown. Green line: regular potential that yields a typical ultrashort soliton. Red line: limiting case of the infinite potential wall. The resulting soliton shows an unphysical cusp. The corresponding pulse duration provides the limiting (the smallest possible) value of the temporal pulse width. Brown line: too short durations lead to singularities, the soliton just does not exist. [21] (b) The shortest soliton (red line) for the Drude dispersion law, $\epsilon(\omega)=1-\omega_{p}^{2} / \omega^{2}$, and the carrier frequency $\omega_{0}=2 \omega_{p}$. There is a pronounced tendency to cusp formation. For comparison a corresponding fundamental soliton is shown (black line). It clearly underestimates the peak power (after [23]).

Solitary solutions of Eq. (1) have been found numerically by successive iterations. First, we analyzed the Drude dispersion law. The tendency to cusp formation was clearly observed (Fig. 1b, red line), the exact shortest soliton significantly differs from the standard fundamental soliton (Fig. 1b, black line). Second, we analyzed solitary solutions for several dispersion laws of real single-mode fibers. Again, we observed a clear tendency to cusp formation in the space-time domain. However, the cusp mechanism competes now with the Cherenkov radiation, the latter may destroy the soliton more quickly than the former.

\section{CONCLUSIONS}

There are several mechanisms that destroy too short optical solitons. Most often this is due to influence of the normal dispersion domain and Cherenkov radiation. However, subcycle solitons were never observed even for the most optimal dispersion profiles. In the absence of Cherenkov radiation another mechanism comes into play. Namely, an unphysical cusp appears at the top of the pulse envelope and the solitary solution is destroyed. This happens at a critical duration of approximately one and half carrier periods.

\section{REFERENCES}

[1] T. Brabec and F. Krausz, "Intense few-cycle laser fields: Frontiers of nonlinear optics," Rev. Mod. Phys., vol. 72, no. 2, pp. 545-591, 2000.

[2] K. Akimoto, "Properties and applications of ultra-short electromagnetic mono- and sub-cycle waves," J. Phys. Soc. Jpn., vol. 65, no. 7, pp. 2020-2032, 1996.

[3] D. V. Skryabin and A. V. Gorbach, "Colloquium: Looking at a soliton through the prism of optical supercontinuum," Rev. Mod. Phys., vol. 82, no. 2, pp. 1287-1299, 2010.

[4] T. Brabec and F. Krausz, "Nonlinear optical pulse propagation in the single-cycle regime," Phys. Rev. Lett., vol. 78, no. 17, pp. 3282-3285, 1997.

[5] J. M. Dudley, G. Genty, and S. Coen, "Supercontinuum generation in photonic crystal fiber," Rev. Mod. Phys., vol. 78, no. 4, pp. 1135-1184, 2006.
[6] G. Genty, P. Kinsler, B. Kibler, and J. M. Dudley, "Nonlinear envelope equation modeling of sub-cycle dynamics and harmonic generation in nonlinear waveguides," Opt. Express, vol. 15, no. 9, pp. 5382-5387, 2007.

[7] H. Leblond and D. Mihalache, "Few-optical-cycle solitons: Modified Korteweg-de Vries sine-Gordon equation versus other non-slowlyvarying-envelope-approximation models," Phys. Rev. A, vol. 79, no. 6, p. $063835,2009$.

[8] A. V. Kim, S. A. Skobelev, D. Anderson, T. Hansson, and M. Lisak, "Extreme nonlinear optics in a Kerr medium: Exact soliton solutions for a few cycles," Phys. Rev. A, vol. 77, no. 4, p. 043823, 2008.

[9] S. A. Skobelev, D. V. Kartashov, and A. V. Kim, "Few-optical-cycle solitons and pulse self-compression in a Kerr medium," Phys. Rev. Lett., vol. 99, no. 20, p. 203902, 2007.

[10] V. G. Bespalov, S. A. Kozlov, Y. A. Shpolyanskiy, and I. A. Walmsley, "Simplified field wave equations for the nonlinear propagation of extremely short light pulses," Phys. Rev. A, vol. 66, no. 1, p. 013811, 2002.

[11] A. I. Maimistov, "Some models of propagation of extremely short electromagnetic pulses in a nonlinear medium," Quantum Electron., vol. 30, no. 4, pp. 287-304, 2000.

[12] A. N. Berkovsky, S. A. Kozlov, and Y. A. Shpolyanskiy, "Self-focusing of few-cycle light pulses in dielectric media," Phys. Rev. A, vol. 72, no. 4, p. 043821, 2005.

[13] M. Kolesik and J. V. Moloney, "Nonlinear optical pulse propagation simulation: From Maxwell's to unidirectional equations," Phys. Rev. E, vol. 70, no. 3, p. 036604, 2004.

[14] M. Kolesik, J. V. Moloney, and M. Mlejnek, "Unidirectional optical pulse propagation equation," Phys. Rev. Lett., vol. 89, no. 28, p. 283902, 2002.

[15] A. V. Kim and S. A. Skobelev, "Few-cycle vector solitons of light," Phys. Rev. A, vol. 83, no. 6, p. 063832, 2011.

[16] P. Kinsler, "Unidirectional optical pulse propagation equation for materials with both electric and magnetic responses," Phys. Rev. A, vol. 81, no. 2, p. 023808, 2010.

[17] S. Amiranashvili, U. Bandelow, and A. Mielke, "Calculation of ultrashort pulse propagation based on rational approximations for medium dispersion," Opt. Quantum Electron., vol. 44, no. 3-5, pp. 241-246, Januar 2012.

[18] N. Akhmediev and M. Karlsson, "Cherenkov radiation emitted by solitons in optical fibers," Phys. Rev. A, vol. 51, no. 3, pp. 2602-2607, 1995.

[19] A. Sakovich and S. Sakovich, "Solitary wave solutions of the short pulse equation," J. Phys. A, vol. 39, no. 22, pp. L361-L367, 2006.

[20] D. V. Kartashov, A. V. Kim, and S. A. Skobelev, "Soliton structures of a wave field with an arbitrary number of oscillations in nonresonance media," JETP Lett., vol. 78, no. 5, pp. 276-280, 2003.

[21] S. Amiranashvili, A. G. Vladimirov, and U. Bandelow, "Solitary-wave solutions for few-cycle optical pulses," Phys. Rev. A, vol. 77, no. 6, p. 063821, June 2008.

[22] S. Amiranashvili, U. Bandelow, and N. Akhmediev, "Dispersion of nonlinear group velocity determines shortest envelope solitons," Phys. Rev. A, vol. 84, no. 4, p. 043834, October 2011.

[23] - "Few-cycle optical solitary waves in nonlinear dispersive media," Phys. Rev. A, vol. 87, no. 1, p. 013805, January 2013. 